\title{
The endocrine disrupting chemical, diethylhexyl phthalate, activates MDR1 gene expression in human colon cancer LS174T cells
}

\author{
Akira Takeshita, Keiji Inagaki, Junko Igarashi-Migitaka', Yasunori Ozawa and Noriyuki Koibuchi \\ Endocrine Center, Toranomon Hospital and Okinaka Memorial Institute for Medical Research, 2-2-2 Toranomon, Minato, Tokyo 105-8470, Japan \\ ${ }^{1}$ Department of Anatomy and Cell Biology, St Marianna University School of Medicine, Kawasaki, Kanagawa 216-8511, Japan \\ ${ }^{2}$ Department of Integrative Physiology, Gunma University School of Medicine, Maebashi, Gunma 371-8511, Japan \\ (Requests for offprints should be addressed to A Takeshita; Email: coactivator@mac.com)
}

\begin{abstract}
Resistance to anticancer drugs is often mediated by the overexpression of P-glycoprotein encoded by the multi-drug resistance (MDR1) gene. The nuclear receptor, steroid and xenobiotic receptor (SXR), is one of the key transcriptional regulators of MDR1 gene expression. A variety of xenobiotics bind to SXR, and stimulate transcription on xenobiotic-response elements (XREs), located in the MDR1 gene promoter. Diethylhexyl phthalate (DEHP) is widely used as a plasticizer for polyvinyl chloride (PVC) medical devices. Previous studies have shown that a significant amount of DEHP leaches from PVC infusion bags and lines during interventions, such as total parenteral nutrition, blood transfusion, and cancer chemotherapy. Thus, the leaching of DEHP during parenteral chemotherapy for cancer patients may facilitate MDR1 expression in various tissues, including cancer cells, which may promote drug resistance. To examine such a hypothesis, the effect of DEHP
\end{abstract}

on SXR-mediated transcription of the MDR1 gene was studied in the human colon adenocarcinoma-derived cell line, LS174T cells, which endogenously express SXR. DEHP increased the SXR-mediated transcription of the MDR 1 gene in luciferase-reporter assays. The induction by DEHP was abrogated when a reporter plasmid containing mutated $\mathrm{DR}+4$ motif in the XRE was used. In a mammalian two-hybrid assay, DEHP recruited steroid receptor co-activator-1 to the ligand-binding domain of SXR. Finally, using real-time reverse transcriptase-PCR, we showed that DEHP increased MDR1 gene expression in a dose-dependent manner. We conclude that DEHP is an inducer of the MDR1 gene in this cell line. As such, the leaching of DEHP from the PVC medical devices may influence the MDR1 expression, which may induce resistance to drugs in certain populations of cancer cells.

Journal of Endocrinology (2006) 190, 897-902

\section{Introduction}

Multi-drug resistance (MDR) is a major problem of cancer chemotherapy. Resistance to anticancer drugs is mainly due to the overexpression of the human MDR1 gene that encodes P-glycoprotein (P-gp), which acts as an ATP-dependent membrane efflux pump to exclude anticancer drugs from tumor cells. Thus, MDR1 decreases intracellular drug concentrations to confer a MDR (for reviews, see Loo \& Clarke 1999, Robert 1999, Gottesman et al. 2002).

The orphan nuclear receptor (NR), steroid and xenobiotic receptor (SXR) (also called pregnane $\mathrm{X}$ receptor), was originally identified as a transcriptional regulator of cytochrome P450 monooxygenase 3A4 (CYP3A4; Bertilsson et al. 1998, Blumberg et al. 1998, Kliewer et al. 1998, Lehmann et al. 1998). A variety of inducers of CYP3A4 bind to SXR as ligands, and stimulate transcription on xenobioticresponse elements (XREs), located in its promoter region (Bertilsson et al. 1998, Blumberg et al. 1998, Kliewer et al. 1998, Lehmann et al. 1998, Goodwin et al. 1999). A recent study revealed that SXR also regulates MDR1 gene expression (Geick et al. 2001, Synold et al. 2001).

Diethylhexyl phthalate (DEHP) is widely used as a plasticizer for polyvinyl chloride (PVC) medical devices such as infusion and lines. The PVC medical devices contain a relatively high percentage $(20-40 \%)$ of DEHP to achieve flexibility, strength, and optical clarity. Since DEHP is lipophilic and not chemically bound to PVC, it can leach out of PVC medical devices. The amount of DEHP leaching depends on the lipophilicity of the fluid in contact with the devices. Thus, substances, such as blood, i.v. lipid emulsion, or total parenteral nutrition (TPN) solution, and surfactants used to solubilize i.v. medications can readily extract DEHP from PVC tubing and containers (for review see Tickner et al. 2001). Anticancer drugs, such as paclitaxel, docetaxel, teniposide, and etoposide are widely used for various chemotherapies, and contain such surfactant as either polysorbate 80 or polyoxyethylated castor oil (Pearson \& Trissel 1993, Trissel 1997, Thiesen \& Kramer 1999). 
Exposure to DEHP has produced adverse effects in laboratory animals (for review see Tickner et al. 2001, Shea 2003). DEHP may cause liver cancers in rodents, and testicular atrophy and disturbance of spermatogenesis in young animals. However, no studies have been performed to evaluate the effects of DEHP exposure in humans. In particular, cancer patients may receive multiple interventions, including cancer chemotherapy, TPN, and blood transfusion, whose treatment may have potential risk of DEHP exposure. We previously reported that DEHP activates human SXRmediated transcription on CYP3A4 promoter (Takeshita et al. 2001). Thus, DEHP may also stimulate the SXR-mediated transcription on MDR1 promoter, particularly within cancer cells, to facilitate MDR.

In the present study, we used the human colon carcinoma cell line LS174T, which endogenously expresses SXR, as a model to elucidate the effect of DEHP on MDR1 expression.

\section{Materials and Methods}

\section{Chemicals and antibodies}

Rifampicin and DEHP were obtained from Sigma Chemical Co. The monoclonal mouse anti-steroid receptor co-activator-1 (SRC-1) antibody (clone SRC01) that was raised against a recombinant human SRC-1 protein was purchased from Lab Vision (Fremont, CA, USA). Clone 29 of a TIF2 monoclonal antibody and clone 34 of a TRAM-1/AIB1 monoclonal antibody were obtained from BD Biosciences Pharmingen (San Diego, CA, USA).

\section{Plasmids}

GAL4 SRC-1-RID, constructed by ligating nuclear receptor interacting domain (RID) containing three LXXLL motifs in human SRC-1 (amino acid residues 595-780) into GAL4 DNA-binding domain (DBD) in the pM expression vector (Clontech), and VP16 SXR-LBD, constructed by ligating the ligand-binding domain (LBD) of human SXR (amino acid residues 107-434) into downstream of the VP16 activation domain in AASV-VP16, were described previously (Takeshita et al. 2002). The luciferase (LUC) reporter construct, $5 \times$ upstream activating sequence (UAS)-thymidine kinase minimum promoter (TK)-LUC was kindly provided by Dr Hollenberg, Beth Israel Deaconess Medical Center, Boston, MA, USA (Cohen et al. 2000). MDR1 promoter fragment $(-7975$ to -7013$)$ containing the cluster of XREs (Geick et al. 2001) was amplified by PCR out of the BAC clone CTB60P12 (Invitrogen; GenBank accession number AC002457) with a forward primer (5'-CGC GGA TCC TCT GCT AGC AGT GTT TCT TGT ATA-3') containing an artificial BamHI site and a reverse primer $\left(5^{\prime}\right.$-TAC GGG GTA CCC ATA TAA GGC AAC TGT TTT GTT-3’) containing an artificial KpnI site. The BamHI/KpnI-digested PCR fragment was ligated between the BamHI/KpnI sites of a LUC gene of a pT109luc vector (Nordeen 1988), which contains the sequence from -109 to +52 of the TK promoter to create MDR1-TK-LUC. To create MUTMDR1-TK-LUC plasmid, site-directed mutagenesis of the $\mathrm{DR}+4$ motif in the MDR1 promoter fragment of MDR1TK-LUC plasmid was performed using the GeneTailor sitedirected mutagenesis system (Invitrogen) according to the manufacture's instructions. The forward primer containing two mutated bases in the center of each half site (mutated bases are underlined) was 5'-CTC TCT CCC AGG AGC AAG AAC AAG TTA GAA CAA TGA GGT AAC- $3^{\prime}$. The overlapped reverse primer was 5'-CTT GCT CCT GGG AGA GAG TTC ATT TGA GAT TAA ACA AG-3'. A plasmid phRL-TK, which contains Renilla LUC gene with the TK promoter, was purchased from Promega.

\section{Transient co-transfection experiments}

The human colon adenocarcinoma-derived cell line LS174T (Tom et al. 1976) was obtained from American Type Culture Collection (Manassas, VA, USA). The cells were grown in phenol red-free Dulbecco's modified Eagle's medium (DMEM) and $10 \%$ fetal calf serum (FCS). The serum was stripped of hormones by constant mixing with 10\% (w/v) AG1-X8 resin (Bio-Rad) and powdered charcoal before ultrafiltration. The cells were maintained without antibiotics. Cells were transiently transfected using a calcium phosphate precipitation method in six-well plates with $2 \mu \mathrm{g}$ reporter plasmid containing MDR1TK-LUC or MUT-MDR 1-TK-LUC, $0 \cdot 2 \mu \mathrm{g}$ human SXR. In some samples, empty expression vector was added to equalize total transfected plasmid concentration. One hundred nanograms phRL-TK plasmid were used as an internal control. Alternatively, co-transfections were performed using $0 \cdot 1 \mu \mathrm{g}$ GAL4 SRC-1-RID and $0 \cdot 5 \mu \mathrm{g}$ VP16 SXR-LBD with $2 \mu \mathrm{g}$ $5 \times$ UAS-TK-LUC. Cells were grown for $24 \mathrm{~h}$ with DMSO vehicle alone or $10 \mu \mathrm{M}$ rifampicin or DEHP, and harvested. Cell extracts were analyzed for luciferase activities by using the Dual-Luciferase Reporter Assay System (Promega) according to the manufacture's instructions. Firefly luciferase activity of reporter plasmid in each sample was corrected by Renilla luciferase activity of control plasmid. The corrected luciferase activities of untreated samples were normalized to the luciferase activities of samples as described in the figures. All transfection studies were repeated in triplicate. The results shown are the mean \pm s.D. $(n=3)$.

\section{Western blot analysis}

Whole-cell extract of LS174T cells was prepared using M-PER protein-extraction reagent (Pierce, Rockford, IL, USA). Clarified extract was obtained by centrifugation at $10000 \mathrm{~g}$. The extract of $20 \mu \mathrm{g}$ was mixed in $20 \mu \mathrm{l} 1 \times$ SDS sample buffer. Then the proteins were separated by SDS/PAGE on a $7 \cdot 5 \%$ minigel and electrophoretically transferred to PVDF membranes. The membranes were blocked with $5 \%$ nonfat dry milk in Tris-buffered saline 
(TBS) buffer (50 mM Tris- $\mathrm{HCl} / 150 \mathrm{mM} \mathrm{NaCl}(\mathrm{pH} \mathrm{7} \cdot 4))$ with $0 \cdot 2 \%$ Tween 20 for $2 \mathrm{~h}$. In Western blotting analysis, the SRC-1, TIF2, and TRAM-1 antibodies were used at 1:200. Immunoreactive bands were detected with SuperSignal Substrate System (Pierce) according to the manufacturer's instructions.

\section{Real-time quantitative RT-PCR}

LS174T cells were grown in phenol red-free DMEM and 10\% hormone-stripped FCS. After 3 days culture, the cells in sixwell plates were treated with the different concentrations of DEHP or rifampicin for $24 \mathrm{~h}$. The mRNA for MDR1 was measured by real-time quantitative RT-PCR using PEApplied Biosystems prism model 7700 (PE-Applied Biosystems, Foster City, CA, USA) sequence detection instrument according to a method of Fujimaki et al. (2002). Total RNA was prepared with RNeasy mini kit (Qiagen) as per the manufacturer's protocol. RNA samples were treated with DNase (Qiagen) to remove any contaminating genomic DNA. The total RNA $(1 \mu \mathrm{g})$ was reverse transcribed in a $20 \mu \mathrm{l}$ reaction mixture containing an oligo-dT primer and M-MLV reverse transcriptase (PE-Applied Biosystems). Reverse transcription was carried out at $25^{\circ} \mathrm{C}$ for $10 \mathrm{~min}, 48^{\circ} \mathrm{C}$ for $30 \mathrm{~min}$, and $95^{\circ} \mathrm{C}$ for $5 \mathrm{~min}$ and incubated at $4{ }^{\circ} \mathrm{C}$. Two microliters cDNA obtained by reverse transcription was amplified in a $50 \mu \mathrm{l}$ reaction mix containing $1 \mu \mathrm{M}$ of each primer and $0 \cdot 25 \mu \mathrm{M}$ TaqMan probe. The sequences of forward and reverse primers were $5^{\prime}$-TGCTCAGACAGGATGTGAGTTG-3' in exons 19 and 20, and 5'-TTACAGCAAGCCTGGAACCTAT- $3^{\prime}$ in exon 21 respectively (GenBank accession number: AC005068 for exons 8-28 of the MDR1). The TaqMan fluorogenic probe used was $5^{\prime}$-FAM-AGCATTGACTACCAGGCTCGCCAATAMRA-3'. For an internal control, we used a TaqMan glyceraldehyde-3-phosphate dehydrogenase (GAPDH) Control Reagent (PE-Applied Biosystems). The thermal cycling was performed with 50 cycles of $95^{\circ} \mathrm{C}$ for $15 \mathrm{~s}$ and $60{ }^{\circ} \mathrm{C}$ for $1 \mathrm{~min}$. The expression of MDR 1 gene was calculated relative to that in untreated cells and normalized for GAPDH mRNA using the $2^{-\Delta \Delta C t}$ method (Livak \& Schmittgen 2001). RNA extraction and real-time PCR were carried out separately for each culture dish. All studies were repeated in triplicate. The representative results shown are the mean \pm s.D. $(n=3)$.

\section{Results}

We used the human colon carcinoma cell line LS174T as a model to elucidate the effect of DEHP on MDR1 gene expression. It has been reported that SXR is endogenously expressed in this cell line and that the human SXR agonist, rifampicin, induces $M D R 1$ gene expression (Geick et al. 2001).

To determine whether DEHP stimulates SXR-mediated transcription of MDR1 gene in LS174T cells, transient transfection assays were performed with a reporter plasmid, MDR1-TK-LUC, containing the DR + 4 of MDR1-XRE, fused to heterologous TK promoter, as well as its mutated $\mathrm{DR}+4$ reporter plasmid, MUT-MDR1-TK-LUC. Rifampicin was used as a positive control for SXR ligand. Previous study showed that SXR cannot form a heterodimer with $\mathrm{RXR}$ on the mutated $\mathrm{DR}+4$ element in electrophoretic mobility shift assay and fails to increase rifampicin-induced transcriptional activation in transfection reporter assay (Geick et al. 2001). As shown in Fig. 1, DEHP, as well as rifampicin, increased the transcription on wild-type MDR1-XRE, suggesting that DEHP binds to endogenous SXR of LS174T cells as a ligand, and then stimulates MDR1 transcription. Co-transfection of SXR enhanced further transcriptional activation. In contrast, the MUT-MDR1XRE did not show a significant induction by SXR, even though SXR was exogenously expressed.

Transcriptional activation by NRs is mediated by liganddependent interaction with co-activators including p160 of the NR co-activator family (SRC-1, TIF2/GRIP1, and TRAM-1/AIB1/RAC3/ACTR; McKenna et al. 1999, Glass \& Rosenfeld 2000). The p160 co-activators bind to a variety of NRs in a ligand-dependent manner and enhance the ligand-induced transcriptional activity. We, thus, examined the expression of these three p160 co-activators in LS174T cells by Western blot analysis. The specificity of monoclonal antibodies used in the present studies has been described elsewhere (Igarashi-Migitaka et al. 2005). The Western blotting of cellular extracts of LS174T cells using each

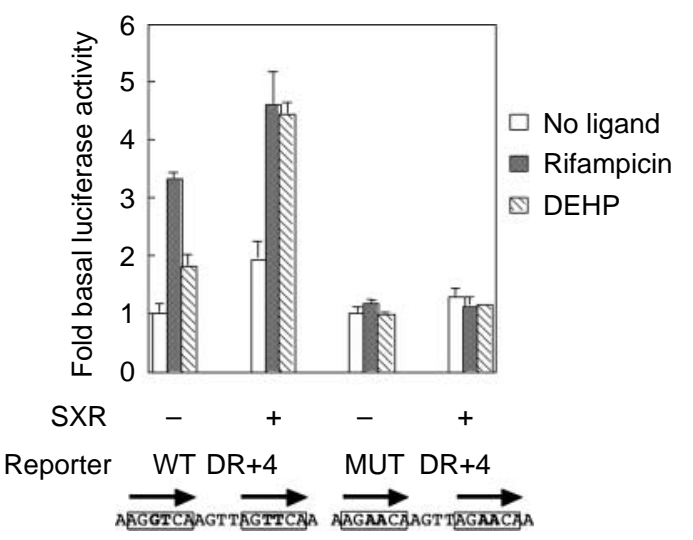

Figure 1 DEHP activates intrinsic SXR-mediated transcription on the MDR1 promoter in LS174T cells. LS174T cells were co-transfected with the reporter plasmid, MDR1-TK-LUC or MUTMDR1-TK-LUC $(2 \mu \mathrm{g}), 0 \cdot 1 \mu \mathrm{g}$ human SXR expression plasmid or empty expression plasmid, and phRL-TK control vector $(0 \cdot 1 \mu \mathrm{g})$. Cells were treated with $10 \mu \mathrm{M}$ rifampicin or DEHP for $24 \mathrm{~h}$ and analyzed for luciferase activity. The firefly luciferase activity from the reporter plasmid was normalized to Renilla luciferase activity from the control plasmid with onefold basal activity defined as the luciferase activity of each reporter in the absence of the ligand and exogenous SXR. The results are expressed as means \pm s.D. $(n=3)$. Open bars, no ligand; shaded bars, rifampicin; hatched bars, DEHP; WT, wild type; MUT, mutant. 
antibody detected a major band at $160 \mathrm{kDa}$ (Fig. 2), indicating that every p160 co-activator is expressed in LS174T cells.

To examine the ligand-induced interaction between SXR and the p160 co-activators, we employed one representative of p160 co-activator, SRC-1 in a mammalian two-hybrid assay. The RID of SRC-1 containing three LXXLL motifs was fused to the DBD of GAL4 (GAL4 SRC-1-RID), and the LBD of human SXR was fused to the transactivation domain of VP16 (VP16 SXR-LBD; Takeshita et al. 2001, 2002). These constructs were co-transfected with a reporter plasmid containing five copies of a GAL4 UAS in LS174T cells. As shown in Fig. 3, GAL4 SRC-1-RID interacted with VP16 SXR-LBD in the presence of rifampicin and DEHP. Thus, DEHP, as well as rifampicin, recruits SRC-1 to the LBD of human SXR in LS174T cells.

To confirm further the induction of MDR1 gene by DEHP, LS174T cells were treated with the different concentrations of DEHP for $24 \mathrm{~h}$, and MDR $1 \mathrm{mRNA}$ levels were determined by real-time quantitative PCR analysis. As shown in Fig. 4, MDR1 mRNA levels in LS174T cells were significantly increased by treatment with DEHP, as well as rifampicin, in a dose-dependent manner. Of note, higher concentrations of DEHP than rifampicin are required for significant induction. DEHP resulted in a $2 \cdot 8$-fold induction of MDR1 mRNA at $10 \mu \mathrm{M}$.

\section{Discussion}

DEHP is an animal carcinogen that can cause fetal death, malformations, and reproductive toxicity in laboratory animals (Tickner et al. 2001, Shea 2003). However, the adverse effects in humans remain uncharacterized. We showed that DEHP acts as a ligand of human SXR to activate MDR1

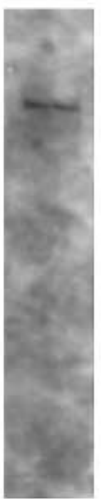

$\mathrm{Ab}$

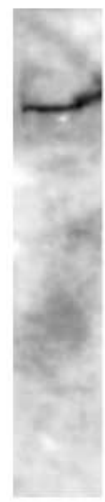

TIF2

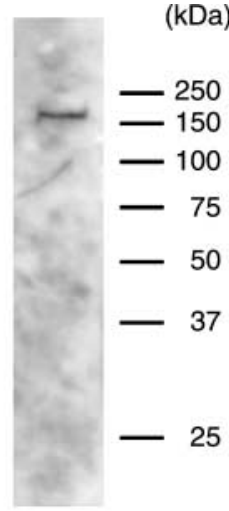

TRAM-1
Figure 2 Expression of p160 steroid receptor co-activators in LS174T cells. Western blot analysis using whole-cell lysates of LS $174 \mathrm{~T}$ cells. Each monoclonal antibody (Ab) detects one major band at $160 \mathrm{kDa}$.

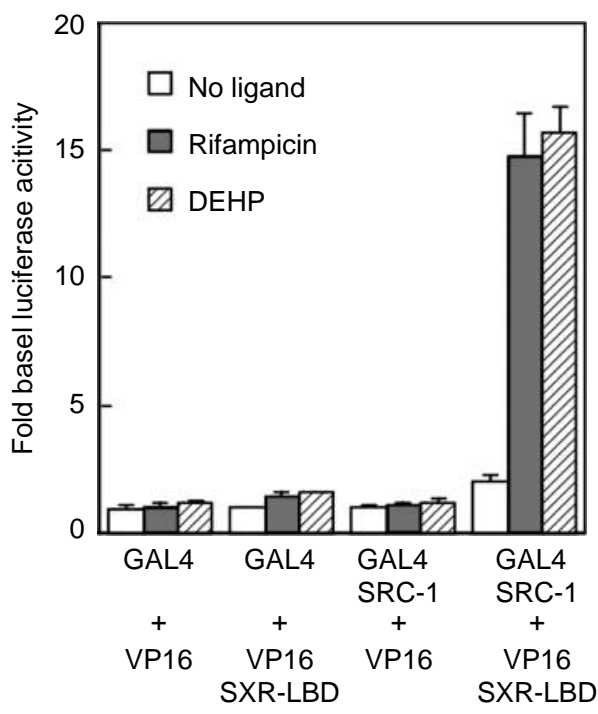

Figure 3 In vivo interaction between LBD of human SXR and RID of SRC-1. The expression plasmids encoding either GAL4 alone or GAL4 SRC-1-RID $(0 \cdot 1 \mu \mathrm{g})$ and either VP16 alone or VP16 SXR-LBD $(0 \cdot 5 \mu \mathrm{g})$ were co-transfected with $5 \times$ UAS-TK-LUC reporter plasmid $(2 \mu \mathrm{g})$ and control vector $(0 \cdot 1 \mu \mathrm{g})$ in LS174T cells. Cells were treated with $10 \mu \mathrm{M}$ rifampicin or DEHP for $24 \mathrm{~h}$. The corrected luciferase activity was calculated as fold luciferase activity with onefold basal activity defined as luciferase activity with GAL4 and VP16 in the absence of ligand. The results are expressed as means \pm s.D. $(n=3)$. Open bars, no ligand; shaded bars, rifampicin; hatched bars, DEHP.

gene of human colon cancer LS174T cells in the present study. DEHP stimulated human SXR-mediated transcription on the XRE of the MDR1 gene promoter. DEHP increased the interaction of LBD of SXR with an NR co-activator,

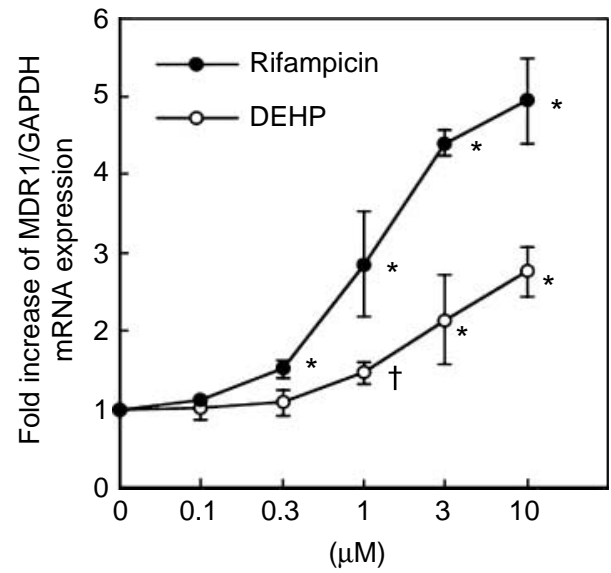

Figure 4 Dose-dependent effect of DEHP on MDR1 gene expression by real-time quantitative PCR analysis. After culture for $48 \mathrm{~h}$ in medium depleted of hormones, LS174T cells were treated with different doses of DEHP or rifampicin for an additional 24-h period. The relative amount of MDR1 mRNAs, compared with that in the cells cultured in the hormone-depleted medium only, is given as fold increase in the ordinates. The expression level of MDR1 mRNA was normalized by GAPDH mRNA. The results are expressed as means \pm S.D. $(n=3) .{ }^{*} P<0 \cdot 005 ;{ }^{\dagger} P<0 \cdot 01$ (versus untreated cells). 
SRC-1. DEHP induced MDR1 mRNA of LS174T cells in a dose-dependent manner. The results have indicated that DEHP may induce local drug resistance in human cancer cells through activation of MDR1 gene via binding to SXR.

A wide variety of xenobiotics can bind to SXR as ligands, and stimulate SXR-mediated transcription. The X-ray crystallographic studies of the LBD of the human SXR revealed that SXR contains a relatively large volume ligand-binding pocket compared with other NRs (Watkins et al. 2001). In addition, the co-crystal structure of SXR with one of its ligands, SR12813 revealed that a single drug molecule was bound in the pocket in three distinct orientations (Watkins et al. 2001). These flexibilities of ligand-binding properties likely enable SXR to recognize a wide range of xenobiotics, including DEHP. As such, DEHP-bound SXR recruits the p160 co-activator, SRC-1, to turn on the switch of MDR1 gene expression.

In normal tissues, SXR is highly expressed in liver and intestine to regulate CYP3A4 genes (Blumberg et al. 1998, Lehmann et al. 1998) and MDR1 (Geick et al. 2001, Synold et al. 2001). Although not all human cancer cells of intestinal and hepatic origin express substantial levels of SXR, it is likely that a significant proportion of these tissue-derived cell lines may express functional SXR. For this reason, we used the human colon cancer cell line LS174T for our investigations. Masuyama et al. (2003) reported functional and significant expression of SXR in endometrial cancer cells, as well. SXR expression in breast cancer has also been documented (Dotzlaw et al. 1999). It will be interesting to study the effect of DEHP on MDR 1 gene expression in such SXR-expressing cancer cells.

Based on reports by other investigators, cancer patients can be exposed to considerable doses of DEHP through certain medical procedures, which may influence MDR 1 expression of tumor cells to induce drug resistance. For instance, approximately $1 \mathrm{mg}$ DEHP is leached from 50 to $80 \mathrm{~cm}$ tubing after infusion of $250 \mathrm{ml}$ containing $100 \mathrm{mg}$ etoposide with a flow rate of $30 \mathrm{ml} / \mathrm{h}$ (Bagel-Boithias et al. 2005). Etoposide contains surfactant, which may extract DEHP from PVC tubing and containers. Other anticancer drugs, such as docetaxel, paclitaxel, and teniposide, as well as various i.v. drugs, including glucocorticoid derivatives, gonadotropinreleasing hormone, vitamins $\mathrm{A}, \mathrm{D}$, and $\mathrm{K}$, granulocyte colony-stimulating factor, erythropoietin, and interferon also contain the same or similar surfactant (Pearson \& Trissel 1993). Cancer patients sometimes receive total TPN as well. The Food and Drug Administration (FDA) estimated that up to $4.4 \mathrm{mg}$ DEHP could be released over a $24 \mathrm{~h}$ period (equivalent to $0.06 \mathrm{mg} / \mathrm{kg}$ per day for a $70 \mathrm{~kg}$ adult) by TPN (Anonymous 2002). An even higher amount of leaching of DEHP by TPN (more than $20 \mathrm{mg} /$ day) was reported (Loff et al. 2000). Chronic transfusion of blood or blood products is also common in the treatment of patients with chemotherapyassociated anemia and blood disorders such as leukemia. DEHP has been measured in blood products (whole blood, plasma, platelet, and packed red cells) at $4-650 \mathrm{mg} / 1$ (Tickner et al. 2001). Furthermore, the mean daily intake of $160 \mu \mathrm{g}$ DEHP was estimated by analysis of hospital diets in Japan (Tsumura et al. 2003). Such estimates did not take into account the exposure of patients from multiple PVC devices. Overall, some cancer patients may be exposed to considerable levels of DEHP.

In response to animal studies demonstrating adverse effects of DEHP exposure to several organs, the FDA has recommended limiting the daily parenteral DEHP exposure to a maximal tolerable intake of $0.6 \mathrm{mg}$ DEHP per $\mathrm{kg}$ body weight (Anonymous 2002). However, this recommendation may not take into account the effect of MDR 1 expression in cancer cells. It has been reported that the mean plasma concentration (with standard error) for DEHP in five normal healthy volunteers is $0 \cdot 62 \pm 0 \cdot 12 \mu \mathrm{g} / \mathrm{ml}$ (i.e. $1 \cdot 6 \mu \mathrm{M}$; Faouzi et al. 1999), consistent with the fact that DEHP is the ubiquitous environmental contaminant (for review see Wams 1987). The mean plasma concentration of DEHP from extraction of $105 \mathrm{mg}$ DEHP from a dialyzer during a hemodialysis session is $1 \cdot 91 \mu \mathrm{g} / \mathrm{ml}$ (i.e. $4 \cdot 9 \mu \mathrm{M}$; Pollack et al. 1985). Since our real time RT-PCR analysis showed that DEHP significantly increased MDR 1 expression at $1 \mu \mathrm{M}$, it may be necessary to further reduce DEHP exposure in patients receiving parenteral therapy.

Blumberg et al. (1998) reported that cocktails of endogenous steroids additively increase human SXRmediated transcription, suggesting that the combination of xenobiotics may additively increase MDR 1 expression. Since anticancer drugs, such as cisplatin (Masuyama et al. 2005) and paclitaxel (Synold et al. 2001), have been known to stimulate SXR-mediated transcription of MDR1 gene, combinations of DEHP with such anticancer drugs may additively activate human SXR. Paclitaxel contains surfactant, polyoxyethylated castor oil, and its manufacturer recommends not using PVCcontaining administration sets to avoid leaching of DEHP. PVC-free medical devices are now readily available in most countries. Our studies suggest the use of PVC-free medical devices for cancer patients may diminish the possibility of drug resistance to cancer chemotherapy.

\section{Acknowledgements}

We are grateful for critical review and editing of the manuscript by Dr P M Yen (Department of Medicine, Johns Hopkins Bayview Medical Center, Baltimore, MD, USA).

\section{Funding}

This study was supported by a grant-in-aid for Scientific Research from the Ministry of Education, Culture, Sports, Science and Technology, Japan. The authors declare that there is no conflict of interest that would prejudice the impartiality of this scientific work. 


\section{References}

Anonymous 2002 Safety Assessment of Di(2ethylhexyl)phthalate (DEHP) Released from PVC Medical Devices: US Food and Drug Administration.

Bagel-Boithias S, Sautou-Miranda V, Bourdeaux D, Tramier V, Boyer A \& Chopineau J 2005 Leaching of diethylhexyl phthalate from multilayer tubing into etoposide infusion solutions. American Journal of Health System Pharmacy 62 182-188.

Bertilsson G, Heidrich J, Svensson K, Asman M, Jendeberg L, SydowBackman M, Ohlsson R, Postlind H, Blomquist P \& Berkenstam A 1998 Identification of a human nuclear receptor defines a new signaling pathway for CYP3A induction. PNAS 95 12208-12213.

Blumberg B, Sabbagh W Jr, Juguilon H, Bolado J Jr, van Meter CM, Ong ES \& Evans RM 1998 SXR, a novel steroid and xenobiotic-sensing nuclear receptor. Genes and Development 12 3195-3205.

Cohen RN, Putney A, Wondisford FE \& Hollenberg AN 2000 The nuclear corepressors recognize distinct nuclear receptor complexes. Molecular Endocrinology 14 900-914.

Dotzlaw H, Leygue E, Watson P \& Murphy LC 1999 The human orphan receptor PXR messenger RNA is expressed in both normal and neoplastic breast tissue. Clinical Cancer Research 5 2103-2107.

Faouzi MA, Dine T, Gressier B, Kambia K, Luyckx M, Pagniez D, Brunet C, Cazin M, Belabed A \& Cazin JC 1999 Exposure of hemodialysis patients to di-2-ethylhexyl phthalate. International Journal of Pharmaceutics 180 113-121.

Fujimaki S, Funato T, Harigae H, Fujiwara J, Kameoka J, Meguro K, Kaku M \& Sasaki T 2002 Quantitative analysis of a MDR1 transcript for prediction of drug resistance in acute leukemia. Clinical Chemistry 48 811-817.

Geick A, Eichelbaum M \& Burk O 2001 Nuclear receptor response elements mediate induction of intestinal MDR1 by rifampin. Journal of Biological Chemistry 276 14581-14587.

Glass CK \& Rosenfeld MG 2000 The coregulator exchange in transcriptional functions of nuclear receptors. Genes and Development 14 121-141.

Goodwin B, Hodgson E \& Liddle C 1999 The orphan human pregnane X receptor mediates the transcriptional activation of CYP3A4 by rifampicin through a distal enhancer module. Molecular Pharmacology 56 1329-1339.

Gottesman MM, Fojo T \& Bates SE 2002 Multidrug resistance in cancer: role of ATP-dependent transporters. Nature Reviews Cancer $248-58$.

Igarashi-Migitaka J, Takeshita A, Koibuchi N, Yamada S, Ohtani-Kaneko R \& Hirata K 2005 Differential expression of p160 steroid receptor coactivators in the rat testis and epididymis. European Journal of Endocrinology 153 595-604.

Kliewer SA, Moore JT, Wade L, Staudinger JL, Watson MA, Jones SA, McKee DD, Oliver BB, Willson TM, Zetterstrom RH et al. 1998 An orphan nuclear receptor activated by pregnanes defines a novel steroid signaling pathway. Cell 92 73-82.

Lehmann JM, McKee DD, Watson MA, Willson TM, Moore JT \& Kliewer SA 1998 The human orphan nuclear receptor PXR is activated by compounds that regulate $C Y P 3 A 4$ gene expression and cause drug interactions. Journal of Clinical Investigation 102 1016-1023.

Livak KJ \& Schmittgen TD 2001 Analysis of relative gene expression data using real-time quantitative PCR and the 2(-Delta Delta $\mathrm{C}(\mathrm{T})$ ) method. Methods 25 402-408.

Loff S, Kabs F, Witt K, Sartoris J, Mandl B, Niessen KH \& Waag KL 2000 Polyvinylchloride infusion lines expose infants to large amounts of toxic plasticizers. Journal of Pediatric Surgery 35 1775-1781.

Loo TW \& Clarke DM 1999 Merck Frosst Award Lecture 1998. Molecular dissection of the human multidrug resistance P-glycoprotein. Biochemistry and Cell Biology 77 11-23.
McKenna NJ, Lanz RB \& O’Malley BW 1999 Nuclear receptor coregulators: cellular and molecular biology. Endocrine Reviews 20 321-344.

Masuyama H, Hiramatsu Y, Kodama J \& Kudo T 2003 Expression and potential roles of pregnane $\mathrm{X}$ receptor in endometrial cancer. Journal of Clinical Endocrinology and Metabolism 88 4446-4454.

Masuyama H, Suwaki N, Tateishi Y, Nakatsukasa H, Segawa T \& Hiramatsu Y 2005 The pregnane $X$ receptor regulates gene expression in a ligand- and promoter-selective fashion. Molecular Endocrinology 19 1170-1180.

Nordeen SK 1988 Luciferase reporter gene vectors for analysis of promoters and enhancers. Biotechniques 6 454-458.

Pearson SD \& Trissel LA 1993 Leaching of diethylhexyl phthalate from polyvinyl chloride containers by selected drugs and formulation components. American Journal of Hospital Pharmacology 50 1405-1409.

Pollack GM, Buchanan JF, Slaughter RL, Kohli RK \& Shen DD 1985 Circulating concentrations of di(2-ethylhexyl) phthalate and its de-esterified phthalic acid products following plasticizer exposure in patients receiving hemodialysis. Toxicology and Applied Pharmacology 79 257-267.

Robert J 1999 Multidrug resistance in oncology: diagnostic and therapeutic approaches. European Jouranl of Clinical Investigation 29 536-545.

Shea KM 2003 Pediatric exposure and potential toxicity of phthalate plasticizers. Pediatrics 111 1467-1474.

Synold TW, Dussault I \& Forman BM 2001 The orphan nuclear receptor SXR coordinately regulates drug metabolism and efflux. Nature Medicine 7 584-590.

Takeshita A, Koibuchi N, Oka J, Taguchi M, Shishiba Y \& Ozawa Y 2001 Bisphenol-A, an environmental estrogen, activates the human orphan nuclear receptor, steroid and xenobiotic receptor-mediated transcription. European Journal of Endocrinology 145 513-517.

Takeshita A, Taguchi M, Koibuchi N \& Ozawa Y 2002 Putative role of the orphan nuclear receptor SXR (steroid and xenobiotic receptor) in the mechanism of CYP3A4 inhibition by xenobiotics. Journal of Biological Chemistry 277 32453-32458.

Thiesen J \& Kramer I 1999 Physico-chemical stability of docetaxel premix solution and docetaxel infusion solutions in PVC bags and polyolefine containers. Pharmacy World Science 21 137-141.

Tickner JA, Schettler T, Guidotti T, McCally M \& Rossi M 2001 Health risks posed by use of Di-2-ethylhexyl phthalate (DEHP) in PVC medical devices: a critical review. American Journal of Industrial Medicine 39 100-111.

Tom BH, Rutzky LP, Jakstys MM, Oyasu R, Kaye CI \& Kahan BD 1976 Human colonic adenocarcinoma cells. I. Establishment and description of a new line. In Vitro 12 180-191.

Trissel LA 1997 Pharmaceutical properties of paclitaxel and their effects on preparation and administration. Pharmacotherapy 17 133S-139S.

Tsumura Y, Ishimitsu S, Saito I, Sakai H, Tsuchida Y \& Tonogai Y 2003 Estimated daily intake of plasticizers in 1-week duplicate diet samples following regulation of DEHP-containing PVC gloves in Japan. Food Additives Contaminants 20 317-324.

Wams TJ 1987 Diethylhexylphthalate as an environmental contaminant - a review. Science of the Total Environment 66 1-16.

Watkins RE, Wisely GB, Moore LB, Collins JL, Lambert MH, Williams SP, Willson TM, Kliewer SA \& Redinbo MR 2001 The human nuclear xenobiotic receptor PXR: structural determinants of directed promiscuity. Science 292 2329-2333.

Received in final form 5 June 2006 Accepted 21 June 2006 